aber, die Konstruktion einer Studie so zu erklären, dass ein Patient das intellektuell verstehe. Vor allem die Ergebnisoffenheit von Studien zu erklären und damit auch die zufällige Zuteilung in Verum- und Kontrollarm, sei eine schwer zu bewältigende Hürde.

Eine weitere Herausforderung stellt die enorme Spezialisierung in der Onkologie dar: die Entdeckung immer neuer Krebs-Entitäten aufgrund neuer molekularbiologischer Erkenntnisse. So werden die Fragestellungen der klinischen Forschung immer spezieller. Damit sinkt die Wahrscheinlichkeit, dass ein bestimmter Patient in eine konkrete klinische Studie eingeschlossen werden kann. Oder anders herum: Es müssen immer mehr Patienten auf ihre Eignung für eine Studie gescreent werden.

Der Aufwand, um eine ausreichend große Studienpopulation zusammenzustellen, steigt also. Diese Screening-Arbeit, die zu einem Großteil niedergelassene Hämatologen und Onkologen leisten, wird nach Angaben von Schmitz von der Industrie nicht bezahlt und beruht im Wesentlichen auf einer intrinsischen Motivation der Ärzte.

Andererseits: Ohne Entgelte der Industrie und andere Quellen wäre die aufwendige Studieninfrastruktur in der ambulanten Onkologie nicht finanzierbar.

\section{Personalintensive Infrastruktur}

Schmitz beschäftigt in seinem Studienzentrum zwei Studien-Koordinatorinnen, die als Studienassistentinnen zertifiziert sind. Im Arzneimittelgesetz ist dieses Berufsbild nicht definiert. In der Praxis ist der Bildungshintergrund mindestens Abitur und Ausbildung zu einem medizinischen Assistenzberuf oder auch ein Studium der Biologie oder der Gesundheitsökonomie. Zwingend nötig sind regulatorische Kenntnisse. Hinzu kommt ein Team von Dokumentationsassistenten, die alle relevanten Daten im Rahmen einer Studie nach präzisen Vorgaben des mit den Zulassungsbehörden abgestimmten Prüfplans erfassen müssen. Meist sind es speziell geschulte Medizinstudenten, die diese Arbeit übernehmen.

Für jede klinische Studie muss aber auch die Studienpraxis ihre Qualifikation nach den Standards der Europäischen Arzneimittelagentur (EMA) und der

\title{
Transparenz durch Kodex
}

Kodizes regeln die Zusammenarbeit der Industrie mit Ärzten, Fachkreisen und Patientenorganisationen. Transparenz wird dabei immer wichtiger.

Ziel des FSA ist die Etablierung von Regeln für die Zusammenarbeit der Pharmaindustrie mit Partnern im Gesundheitswesen: Diese Regeln binden die Firmen, nicht die Ärzte. Die Mediziner entscheiden selbst, ob und wie sie mit der Industrie zusammenarbeiten und wie transparent sie das machen wollen.

Der FSA ist der Verein „Freiwillige Selbstkontrolle für die Arzneimittelindustrie e.V.“ und wurde 2004 von den Mitgliedern des Verbandes forschender Pharmaunternehmen (vfa) gegründet. Jedes vfa-Mitglied muss auch Mitglied des FSA sein. Weitere Unternehmen haben sich angeschlossen.

Der FSA überwacht diese Firmen in der korrekten Zusammenarbeit mit Ärzten und anderen Angehörigen der medizinischen Fachkreise. Die FSA-Mitglieder haben sich bislang zu drei Kodizes verpflichtet: dem Kodex für die Zusammenarbeit mit Fachkreisen, dem Kodex zur Zusammenarbeit mit Patientenorganisationen sowie dem Transparenzkodex. Erster Schritt war 2004 der FSA-Kodex für die Zusammenarbeit mit Fachkreisen. 2008 folgte der Kodex für die Kooperation mit Patientenorganisationen. 2013 hat der FSA mit dem Transparenzkodex seine Mitglieder verpflichtet, ihre Leistungen an Ärzte und andere Fachkreisangehörige transparent zu machen. Der FSA folgt damit einem Beschluss des europäischen Pharmaverbandes EFPIA. Die Erstveröffentlichung für 2015 erfolgte zur Jahresmitte 2016. Danach haben Ärzte, Fachkreisangehörige sowie medizinische Einrichtungen und Organisationen insgesamt 575 Millionen Euro aufgrund ihrer Leistungen von der Industrie erhalten, und zwar:

366 Millionen Euro für Forschung wie zum Beispiel klinische Studien,

- 119 Millionen Euro für Vortragshonorare oder Kostenerstattungen für Fortbildungen, 90 Millionen Euro für die Unterstützung von Veranstaltungen, Organisationen und Institutionen. Helmut Laschet

amerikanischen Zulassungsbehörde FDA nachweisen. Schon im Vorfeld des Qualifikationschecks entsteht hierbei ein Korrespondenzvolumen von 50 Emails zur Darlegung der Praxisstrukturen und der Rekrutierungsfähigkeit von Patienten. Sind die Voraussetzungen erfüllt, müssen zwischen dem Sponsor der Studie, also einem pharmazeutischen Unternehmen, oder einer Contract Research Organisation, die die Studie im Auftrag des Arzneimittelherstellers durchführt, und der Studienpraxis ein Vertrag über Leistungen und Honorare abgeschlossen werden. Vor dem Hintergrund des hohen Aufwands empfiehlt Schmitz dringend, die Wirtschaftlichkeit einer solchen Studie für die Praxis zu prüfen, um nicht in die roten Zahlen zu geraten. Allein diese Vorlaufphase dauert etwa ein Jahr. Und durchaus zwei Jahre kann es dauern, bis die vorgesehene Zahl an Probanden rekrutiert ist.

\section{Versorgung auf Uni-Niveau}

In der Prüfphase selbst gelten strikt vorgegebene wechselseitige Monitoringund Reportpflichten. Das heißt: In Stu dien eingeschlossene Patienten werden diagnostisch besonders intensiv und planmäßig überwacht, nicht zuletzt, um unverzüglich auftretende Risiken zu erfassen und zu bewerten. Vice versa muss der Prüfarzt sogenannte SUSAR-Berichte aktuell monitoren: Darin informieren die Zulassungsbehörden über Ereignisse, die Prüfärzte ihrerseits zu melden verpflichtet sind. Je nach Schweregrad der Nebenwirkung ist eine Meldefrist von einem bis zu vier Tagen vorgesehen. Angesichts dieses hohen Aufwandes für Zeit und Infrastruktur spielt die wirtschaftliche Motivation zur Teilnahme an Studien eine untergeordnete Rolle.

Viel wichtiger, so Schmitz, ist es, Player in der wissenschaftlichen Kommune zu sein, in engem Austausch mit den universitären Behandlungszentren zu stehen und Innovationen auch in die ambulante Praxis zu transferieren. Unter diesen Bedingungen sei es gelungen, auch in der ambulanten Versorgung eine Qualität auf Universitätsniveau zu erreichen.

Helmut Laschet

Dieser Beitrag ist eine Sonderpublikation und erscheint mit freundlicher Unterstützung des Verbandes forschender Pharmaunternehmen (vfa). 